See discussions, stats, and author profiles for this publication at: https://www.researchgate.net/publication/279191742

\title{
Immersive and Interactive Simulator to Support Educational Teaching
}

Conference Paper · June 2015

DOI: 10.1007/978-3-319-21413-9_18

\section{CITATIONS}

0

5 authors, including:

Marcelo De Paiva Guimaraes

Universidade Federal de São Paulo

113 PUBLICATIONS 152 CITATIONS

SEE PROFILE

Valéria Farinazzo Martins

Universidade Presbiteriana Mackenzie

69 PUBLICATIONS 130 CITATIONS

SEE PROFILE

\section{READS}

60

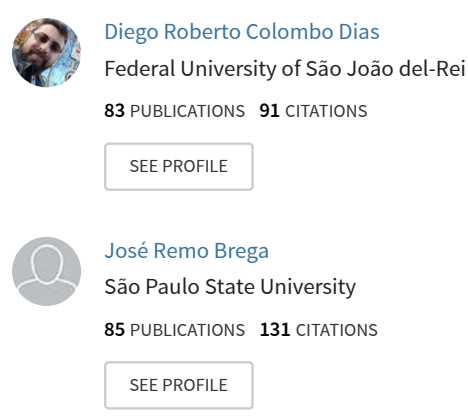

Some of the authors of this publication are also working on these related projects:

Uso da Realidade Virtual na reabilitação de pacientes com AVC e avaliação utilizando conectividade cerebral View project

Objetos de aprendizagem interativos: conceito, ontologia e uso View project 


\title{
Immersive and Interactive Simulator to Support Educational Teaching
}

\author{
Marcelo de Paiva Guimarães ${ }^{1,2}$, Diego Colombo Dias ${ }^{3}$, Valéria Farinazzo Martins ${ }^{4}$, \\ José Remo Brega ${ }^{5}$, Luís Carlos Trevelin ${ }^{3}$ \\ ${ }^{1}$ Universidade Aberta do Brasil, UNIFESP, São Paulo, SP Brasil \\ ${ }^{2}$ Programa de Mestrado em Ciência da Computação, FACCAMP, Campo Limpo Paulista, \\ SP, Brasil \\ ${ }^{3}$ Universidade Federal de São Carlos, UFSCAR, São Carlos,SP - Brasil \\ ${ }^{4}$ Faculdade de Computação e Informática, Universidade Presbiteriana Mackenzie, São \\ Paulo, Brasil \\ ${ }^{5}$ Universidade Estadual Paulista "Júlio de Mesquita Filho", Bauru, SP, Brasil \\ \{marcelodepaiva, diegocolombo.dias, valfarinazzo, remobre- \\ ga\}@gmail.com,trevelin@dc.ufscar.br
}

\begin{abstract}
Visualization and manipulation of a discipline's content integrated with educational planning can enhance teaching and facilitate the learning process. This paper presents a set of tools developed to explore educational content using a multi projection system with high immersion and interaction support for group learning and a support run on Internet browsers for individual learning. The objects visualized are enriched with multimedia content such as video, audio, and text and can be used in different educational proposals. In this work, these tools were populated with content for teaching computer architecture for computer undergraduate students.
\end{abstract}

Keywords: Virtual Reality, Immersive Visualization, Teaching, Learning, Multimedia.

\section{Introduction}

It is becoming increasingly common for students in educational environments to use smartphones, laptops, and tablets with high processing capacity and visualization. This has motivated studies on integrating these devices with educational practices in classrooms, which might allow more effective support for the new digital culture age. Teachers need to understand the modern technological society and how this current digital culture can be favorable to the learning process, bringing interactivity and autonomy to the process of knowledge acquisition.

At present, teachers can take advantage of digital technologies in educational practices, promoting real knowledge gain according to the level of development, skills, and abilities of students in training [1]. Therefore, mere technology adoption is not enough; this requires planning and appropriate tools. The main features of the digital learning culture point to new needs in student training [2, 3, 4, 5, 6], for example, how to organize and assign meaning and sense to information researched by 
learners, in order to build knowledge and develop the capacity of learning management, knowledge, and training.

Simulation is an example of a technological tool that can be used by teachers to get closer to the actual digital culture age. By means of this, it is possible to represent situations that would be impossible without this computational solution, even in real laboratories [7, 8]; one example is an expedition inside an erupting volcano [9]. Using simulators, the users enter a virtual place and visualize, manipulate, and exploit some data in real time [10]. For that, they use their senses, particularly the natural threedimensional movements of the body. A simulation activity can strengthen, facilitate, and consolidate the understanding of involved concepts during a study. Moreover, such activities must be supported by a well-defined pedagogical strategy to be able to achieve good results in the educational process. Simulation can also be a precious tool in group work, mainly in situations that involve decisions during an activity such as, for example, choosing the route in a driving simulation. Different students can test and evaluate various hypotheses and thus understand their decisions and those decisions' consequences. Moreover, adoption of simulators in a classroom tends to encourage and promote the learning process, making it an exciting way of learning.

There are several kinds of simulators [11,12], for instance, static simulators, which requires no whole-body movement or significant physical effort by the students; another example is dynamic simulators, which the users move through the immersive and interactive virtual environment, providing a greater degree of freedom. The immersive simulators discussed in this paper are dynamic, and they are based on virtual reality, a computer area able to create synthetic environments that enable the exploration of basic senses of the human body such as vision, hearing, touch, and smell. The main features of these tools are immersion, interactivity, and involvement. Immersion has the capacity to hold the user's attention; interactivity is related to the capacity of the system to respond to user actions; and the involvement is aimed at user engagement in the activity. User immersive interaction is achieved, for instance, by using 3D stereoscopic display devices, touch feeling devices (haptic devices), and devices that capture the user's movements. User engagement with simulations is achieved with planned actions during the simulation, for example, setting challenges to be achieved.

This paper presents a set of educational tools developed to support well-defined pedagogical strategies that promote learning situations that are not arbitrary. The first tool is an immersive and interactive simulator running on a multi projection system, a CAVE-like (Cave Automatic Virtual Environment [13]) system called miniCAVE. In this environment, teacher and students work in a group to simulate some educational content. The other tool shown is also a simulator, but it runs on web browsers (e.g., Firefox, Chrome, and Explorer), and it is aimed at promoting student self-study. It is also part of the set of two other tools that are used for the preparation of the simulation.

The paper is organized as follows: section 2 discusses the use of simulations in teaching; section 3 presents the research method; section 4 presents the developed computational tools; section 5 describes the process of creating educational content for these tools; and finally, section 6 covers some conclusions. 


\section{Using Simulations for Teaching}

The use of simulators in the educational context is not new; it has already been applied in various situations such as the teaching of physics [14], chemistry [15], biology [16, 17], and medicine [18, 19]. Guimarães and Gnecco [20] showed virtual environments for astronomy education. Dias and colleagues [21] developed a system to support the teaching of dental structures using semantic descriptions in virtual environments. Souza and colleagues [22] have created a simulator for the operating systems discipline.

The tools presented in this paper are different from the others in several aspects, including: the high degree of immersion (3D) and interaction by means of input devices such as Kinect, the Wii remote, a keyboard, and a mouse; the possibility of developing activities in groups or individually; customization that addresses educational content, and integration with multimedia resources (video, audio, and text). The support that allows the semantic description (meaning) of the models is part of such tools. This feature makes the organization of the content flexible; for example, in a computer motherboard simulation, the teacher can simply select the processor and can easily assign it (through links) with some multimedia content available on the Internet. Thus, the developed tools described in this paper go beyond a simple viewer of 3D objects; they are a solution that allows a teacher to plan and execute lessons according to his or her educational goals.

Until recently, the effective use of virtual reality applications in the educational context had as barriers the high price of the equipment and the small amount of available software. However, technological advances have reversed this situation; current$1 \mathrm{y}$, it is possible to build virtual reality laboratories at an affordable cost for educational institutions [23]. Equipment such as computers and projectors are capable of generating good quality images, and appropriate software can now be purchased in the marketplace [20].

The tools presented in this paper provide the use of high quality and easy to use simulations in the educational context. The goal is to provide solutions that allow teachers to focus their work on educational goals and not on technological aspects.

Figure 1 depicts the use of our tools. Initially, the content is addressed by the teacher; afterward, the teacher defines the educational strategy and content and then performs the group simulation (at an institution). After this, the students perform their self-study (at home). And finally, the learning is evaluated and is used as input into a new teaching-learning cycle. Thus, it is expected that students will play the role of coauthors of the learning situations by means of scenario simulations guided by immersion, interactivity, and involvement. Viewing scenarios should be able to stimulate interest and, consequently, enhance the learning activity [1]. 


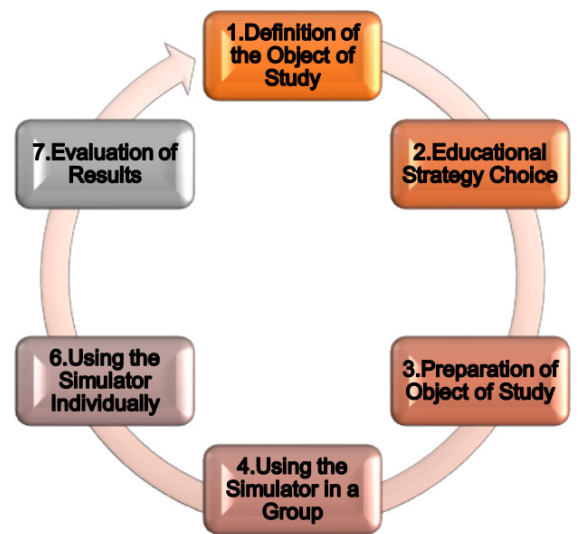

Fig. 1. Teaching-learning cycle

The success of learning to use our tools depends on this cycle, which requires a clear definition of goals and steps taken during the use of the resources involved. For this, additional complementary resources such as formal presentations, readings, and discussions are required in the classroom. Only in this way can education become efficient and effective, enabling the student to apply such knowledge in the real world.

\section{Research Method}

Most software for immersive and interactive environments is designed to display 3D models and is generally tailored to specific problems, for instance, astronomy or physical education. So we projected the tools from the beginning to be adapted to various educational contexts. For this, we used Unified Modeling Language (UML). The agile method of extreme programming [24, 25] was used to develop the set of tools. The teamwork was done by the authors of this paper, who are from the computer and education areas. The tools were developed using the Java language, which allowed the development of web and local tools in a similar way, since Java applications are able to run on browsers (as applets). Finally, we used the content of the computer architecture discipline to perform the validation.

\section{Using Simulations for Teaching-Learning Process}

This section presents the developed tools, which are:

- Immersive viewing: this is a tool used by the teacher to provide 3D immersion and interaction with objects during a group activity. It is executed on multi projection systems, such as CAVE-like systems. This tool depends on specific equipment such as screens and projectors, so it is best executed in an educational institute that has a virtual reality laboratory. It runs on a commodity cluster that has 3D graphic cards. 
- Simple viewing: this is a tool used by students to perform self-study. It runs on Internet browsers, so it can run on a personal computer.

- Multimedia editor: this is a tool used by the teacher that allows the association of multimedia content with 3D models.

- Semantic editor: this is a tool used by the teacher to create semantic descriptions about every 3D model visualized.

\subsection{Immersive Viewing}

Figure 2 depicts the Immersive Viewing tool being executed. In this example, the application is running in a miniCAVE environment composed of three side walls with passive stereoscopic 3D and supporting multiple interaction devices. To ensure that everyone can interact satisfactorily with the environment, it should have a maximum of 12 people using it.

In addition to the polarized screens, the environment consists of a six-node computer cluster that has 3D graphics cards, six high-definition projectors, and interaction devices such as Microsoft Kinect, Wiimote, and keyboard/mouse. There are three kinds of nodes: the Coordinator node that guarantees the distribution and synchronization of information; the Interface node that receives the interactions of users, for example, entries captured from a Microsoft Kinect; and the Output nodes, which process the images and send them to the projectors. This division of functionality allows the assigning of tasks according to each node's characteristics; for example, a mobile device, which has low processing capacity, can be responsible for the acquisition of user interaction data.

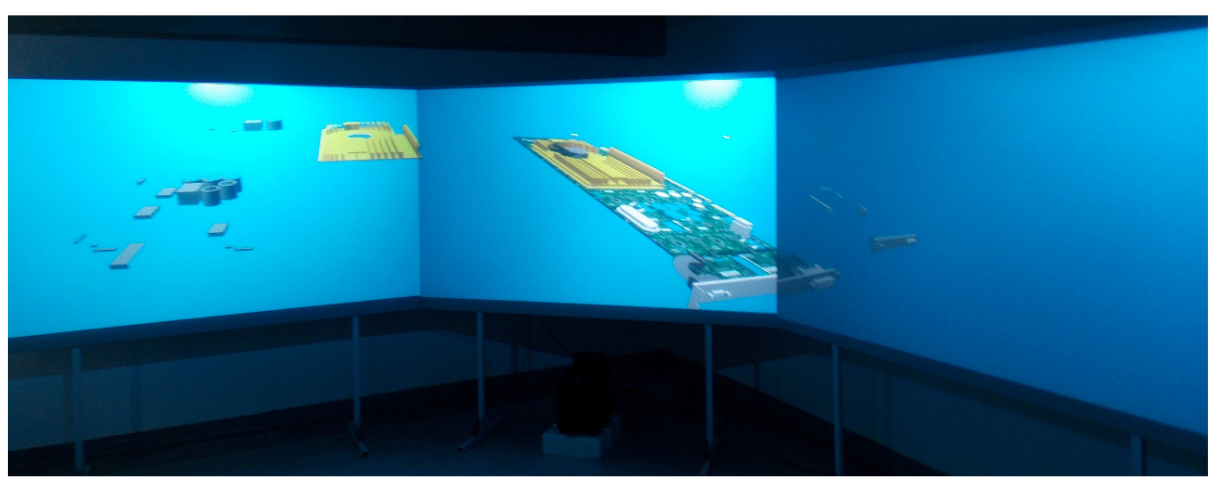

Fig. 2. Computer architecture class - video card

Besides offering immersion, this tool has multimodal support for interaction. Teachers and students can navigate freely in the environment, with the opportunity to choose what they want to view and interact with. The user interaction generates changes to the application state, generating feedback. For example, if the student tries to fit some component into a wrong slot, the component becomes red to alert them. The users can examine the structure and properties of the 3D objects. No special knowledge is required for users to manipulate the interface.

The mounting cost of a display laboratory as shown in Figure 2 is low when compared with the most used solutions in the past decade [20]. However, some stud- 
ies argue that it is still impossible for some institutions to have these kinds of environments, since they still involve necessary equipment, trained teachers, applications, and physical space. Considering this context, it is possible to simplify it; for example, the visualization can be performed in a classroom using just one projector.

\subsection{Simple Viewing}

The Simple Viewer (Figure 3) allows students to run simulations on browsers on their own personal computers. It was designed to run in a Web environment, aiming to omit all installation steps by the user. Thus, the user needs only a browser to visualize and manipulate the 3D virtual models presented in the classroom.

It is possible to visualize the same content in the Immersive Viewer, but with limited interaction resources, immersion, and involvement. For example, this tool only supports anaglyph stereoscopy, and the execution of associated videos should be available in a web video server.

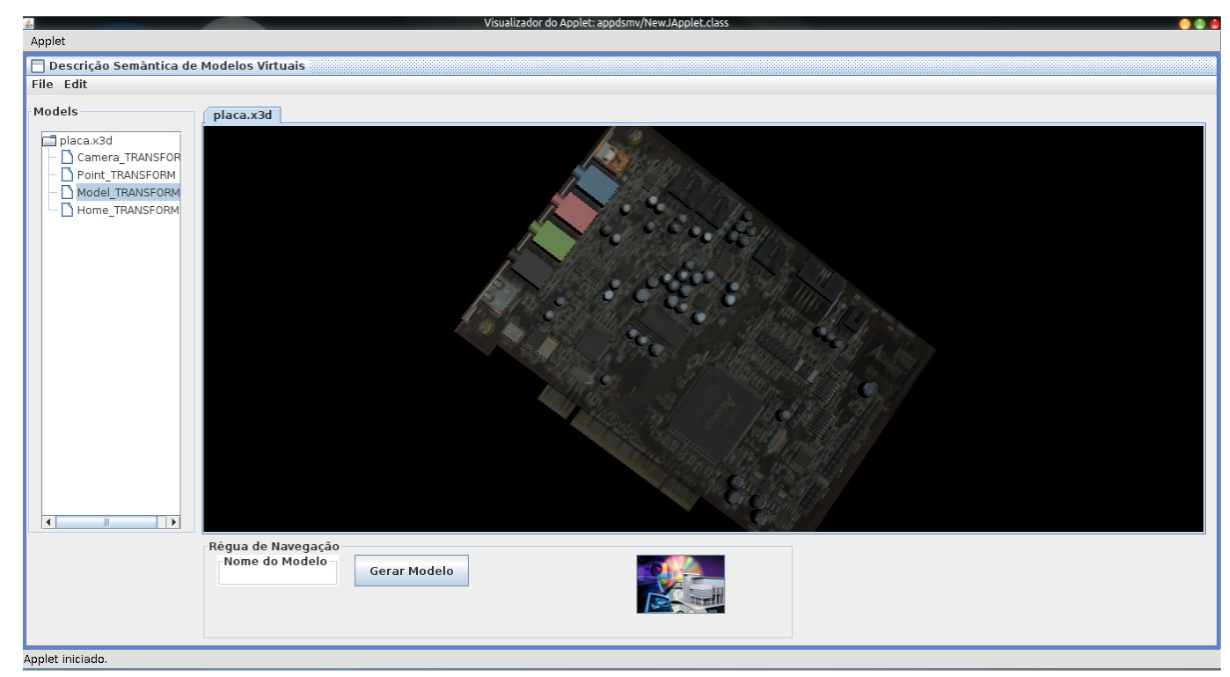

Fig. 3. Simple Viewer running on a browser

\subsection{Multimedia Editor}

The multimedia content editor is associated with each part of the displayed mod$\mathrm{el}$, as is depicted in Figures 2 and 3. Using this, for example, the teacher can associate a video to a capacitor. When the capacitor is selected, the video is displayed. Thus, planning lessons should take into account the object and the multimedia content previously associated with it.

The model can be decomposed into parts, for example, a video card 3D model, for a given class. The teacher can determine that the class will only be addressed with a GPU (graphics processing unit), which allows the flexible development of the class. Thus, the same video card 3D model can be used with various approaches. 
The 3D models must be created in a decomposed form or in shaped parts to allow the teacher to plan a class according to a specific subject. The semantic description tool takes advantage of this decomposition to describe the model and to separate the objects into parts. Thus, the teacher is free to tailor the displayed content to the learning content, including changing the multimedia content associated with the class focus. The system supports different content types such as presentation (ppt, pps, and pptx), text (txt, pdf, doc, docx, and html), image (jpg, bmp, gif, and png), and video (avi, mov, jpeg, and wmv). The objects are stored in X3D format (Extensible 3D Graphics).

\subsection{Semantic Editor}

The Semantic Editor promotes the use of description in 3D environments, using metadata to select, extract semantic objects, and generate queries. This creates a vocabulary of terms that facilitate discovery, retrieval, and integration among objects. This feature makes easy the reuse of 3D models. For this purpose, the Dublin Core Metadata model was used, which is composed of 15 optional properties to describe the object, such as title, creator, format, identifier, origin, language, relation, coverage, and rights.

Figure 4 depicts the Semantic Editor interface. With organized objects, the process of planning and constructing classes is facilitated. The semantic description can help the teachers to find other models (submodels) and types of content that are available on the Internet via a search engine.

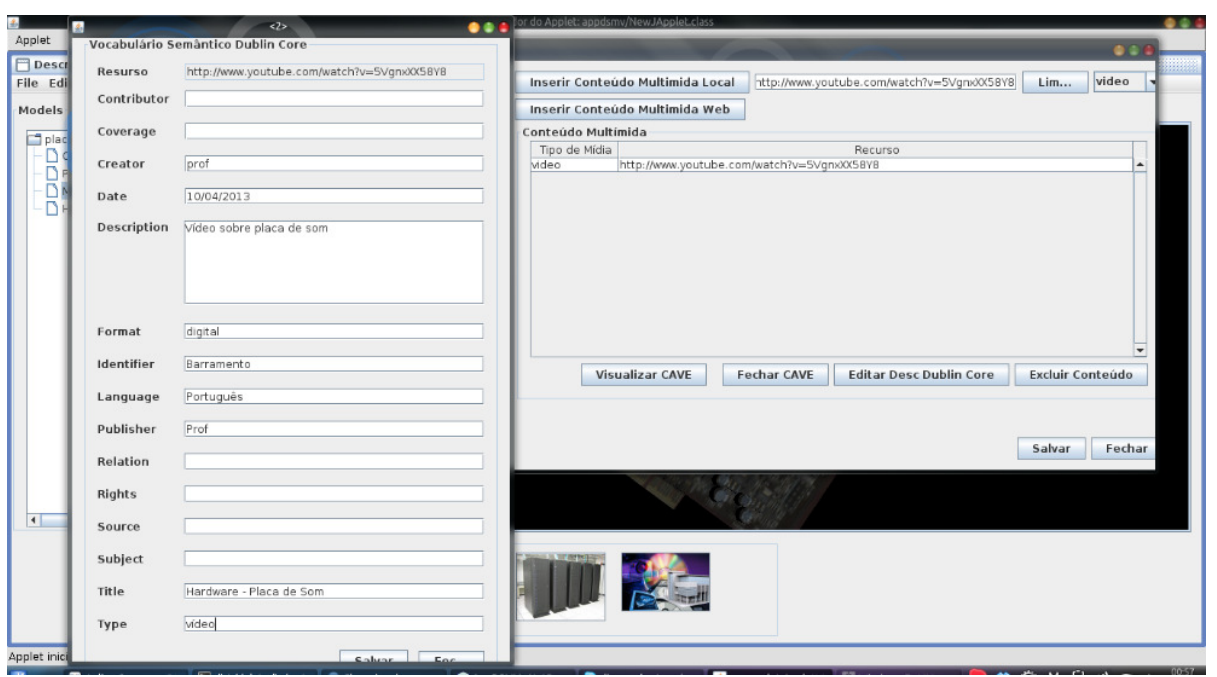

Fig. 4. Content description and aggregation of parts of a 3D model 


\section{Creation of Content}

Before creating some 3D models, it is necessary to define the educational goals that will permeate the teacher education plan, following the educational strategies associated with the potential and limitations of the tools. Figure 5 depicts an example of a strategy that we developed for the computer architecture discipline for undergraduate students in courses related to computing. Initially, the teacher sets the objective content, which is the understanding of the functioning of the main parts of a video card, and sets the strategy to be used, consisting of the following: 1 . Present to the classroom the main parts of a video card; 2. Use Immersive Viewing, which allows the mounting of a virtual video card by teacher and students; 3. Use Simple Viewing, where each student can conduct self-study about the video card and can access the associated multimedia content with it (video and text); 4. Execute an evaluation of the learning content.

\begin{tabular}{c|c|c|c|c|}
$\begin{array}{c}\text { 1. Presentation } \\
\text { of concepts in } \\
\text { classroom }\end{array}$ & $\begin{array}{c}\text { 4. Evaluantion } \\
\text { of the learning } \\
\text { Viewing } \\
\text { content }\end{array}$ \\
\hline
\end{tabular}

Fig. 5. Case of study - computer architecture

We chose to use these tools to teach the computer architecture discipline because the viewers allow the simulation of concepts whose viewing is not possible in the real world, such as the energizing process of a computer motherboard, performed via an animation model. It should be emphasized that the tools are prepared to work with any $3 \mathrm{D}$ content, that is, they are not linked to a specific discipline.

With the teaching objectives and technical-pedagogical strategies defined, the creation of 3D models is the next phase (Figure 6). In phase 1, a technological evaluation of the feasibility of the simulation occurs, which is crucial for the creation of $3 \mathrm{D}$ models. For example, it takes into account the available computing resources (computers, projects, CAVEs, or others) and whether the model will be activated or not. In phase 2, template creation, two approaches are possible: the modeling of 3D objects or the use of models already created.

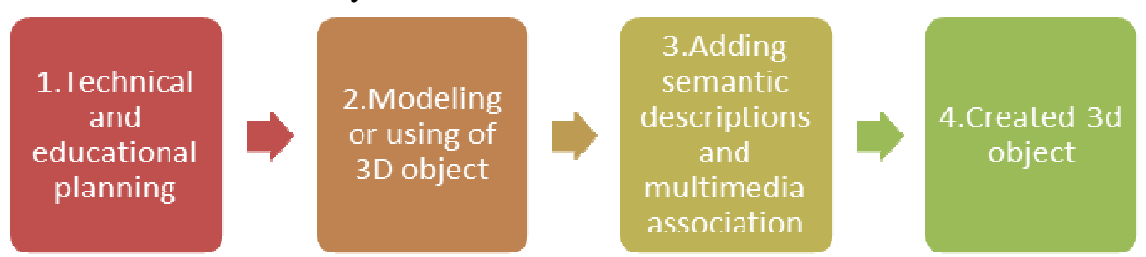

Fig. 6. Process of educational content generation

The advantage of modeling objects is that every member of the teaching staff involved can determine how the objects should be, but this kind of modeling requires more time to run (even months), and a technical team of modelers is required. On the other hand, when a ready $3 \mathrm{D}$ object is obtained, the educational content should be designed in accordance with its predetermined characteristics; however, the time at this stage will be spent in finding the right objects, which may or may not happen.

A crucial and difficult point to solve is when a teacher needs a crew to perform the $3 \mathrm{D}$ modeling of objects, since 3D objects, whether free or paid, may not be suffi- 
cient for the pedagogical approach adopted. This modeling takes time and makes the teacher dependent on such services. However, the trend is that in the future, there will be more $3 \mathrm{D}$ objects available on the Internet, since the number of 3D object repositories has been increasing. In phase 3 , the teacher and/or technical staff add the semantic description to the objects, performing association (creating links) with the multimedia content. Finally, the 3D models are created in step 4.

The generation of an effective range of $3 \mathrm{D}$ content models linked to educational content is a challenge for everyone, because several hurdles must still be overcome, such as the identification of educational content that can take advantage of an environment such as that developed in this project. This challenge exists because teachers do not yet know enough about the potential of immersive and interactive simulation, and there are few user-friendly tools. Another barrier that limits the advance in the use of these simulations is the gap between the specifications of content and preparation. In addition, even if the cost of implementing these simulations is lower than solutions of the past decade, institutions still need the financial viability to incorporate this educational context.

\section{Conclusions}

There is interest in educational simulations that facilitate the teaching-learning process. However, an effective use of simulators based on virtual reality that is affordable and easy to use must be provided. This requires a solution where the teacher him-/herself can draw up all the simulations simply based on content. But the fact is that the everyday use of technology in the classroom is not so simple, because it may often fail, and it requires specific training and much time to prepare content for it. Ideally, the teacher's lesson preparation time should be spent primarily on educational planning and educational content and not on technical issues such as 3D object modeling. However, the scenario has changed in recent years, and more and more teachers can use these resources without major problems. For example, Google Maps is a virtual map that can easily be used by geography teachers; it is enough just to have an Internet connection to use it.

This paper presented a set of tools to facilitate the simulation of educational content in an easy and flexible way. We developed the following: Immersive and Interactive Viewing, Simple Viewing, a Multimedia Editor, and a Semantic Editor. With these tools, it is expected that teachers and students can view and interact with 3D models, enhance the understanding of content, and stimulate learning within the new digital culture learning context. It is important to highlight the ease of content creation, as it is a key factor in using this tool effectively in the classroom, since the teacher should spend his/her time on the pedagogical rather than on the technological issues. In addition, the flexibility of its use should also be stressed, since this tool can be run on a browser or even on CAVE-like systems.

In future research, we plan to improve the tool set, adding new features such as support for Augmented Reality content. We also plan to add new content and perform evaluations of the tool set's use in different courses. 


\section{References}

1. L. S. Vygotsky. The problem of age (m. hall, trans.). R. W. Rieber, 5:187-205 (1998).

2. B. H. J. E. Greenhow, Christine; Robelia. Learning, teaching, and scholarship in a digital age: Web 2.0 and classroom research - what path should we take "now"? Educational Researcher, 38(4):246-259,(2009).

3. K.-W. Lai. Digital technology and the culture of teaching and learning in higher education. Australasian Journal of Educational Technology, 27(Special issue, 8):1263-1275, (2011).

4. R. Saljo. Digital tools and challenges to institutional traditions of learning: technologies, social memory and the performative nature of learning. Journal of Computer Assisted Learning, 26(1):53-64, (2010).

5. D. Thomas and J. S. Brown. A New Culture of Learning: Cultivating the Imagination for a World of Constant Change. CreateSpace Independent Publishing Platform, (2011).

6. S. Wang, H. Hsu, T. Reeves, and D. Coster. Professional development to enhance teachers' practices in using information and communication technologies (icts) as cognitive tools: Lessons learned from a design-based research study. Computer Education, 79:101-115, (2014).

7. M. Amirian, S. Lindner, E. Trabulsi, and C. Lallas. Surgical suturing training with virtual reality simulation versus dry lab practice: an evaluation of performance improvement, content, and face validity. Journal of Robotic Surgery, 8(4):329-335, (2014).

8. S. Smith and E. Ericson. Using immersive game-based virtual reality to teach are-safety skills to children. Virtual Reality, 13(2):87-99, (2009).

9. H. Boudreaux, P. Bible, C. Cruz-Neira, T. Parham, C. Cervato, W. Gallus, and P. Stelling. V-volcano: Addressing students misconceptions in earth sciences learning through virtual reality simulations. In G. Bebis, R. Boyle, B. Parvin, D. Koracin, Y. Kuno, J. Wang, J.-X. Wang, J. Wang, R. Pajarola, P. Lindstrom, A. Hinkenjann, M. Encarnao, C. Silva, and D. Coming, editors, Advances in Visual Computing, volume 5875 of Lecture Notes in Computer Science, pages 1009-1018. Springer Berlin Heidelberg, (2009).

10. T.W. Kuhlen, B. Hentschel. Quo Vadis CAVE: Does Immersive Visualization Still Matter? Computer Graphics and Applications, IEEE, vol.34, no.5, Sept.-Oct, pp.14-21 (2014).

11. B. Aykent, F. Merienne, C. Guillet, D. Paillot and A. Kemeny. Motion sickness evaluation and comparison for a static driving simulator and a dynamic driving simulator. Proceedings of the Institution of Mechanical Engineers, Part D: Journal of Automobile Engineering, 1-2, (2014).

12. B. J. Jaeger and R. R. Mourant R. Comparison of Simulator Sickness Using Static and Dynamic Walking Simulators, Proceedings of the Human Factors and Ergonomics Society 45th Annual Meeting, October, 1896-1900, (2001).

13. C. Cruz-Neira and D. J. Sandin, The CAVE: audio visual experience automatic virtual environment, Magazine Communications of the ACM, Volume 35 Issue 6, June, 64-72, (1992).

14. L. R. Franco, E. Raimann, E., R.R. de Souza and M.W. de Souza Ribeiro, Force and Motion: Virtual Reality as a Study Instrument of Alternative Conceptions in Dynamics, XIII Symposium on Virtual Reality (SVR),23-26 May 2011, Uberlandia, Brazil, 89 - 95, (2011).

15. P. Sampaio, R. Mendonça and S.a Carreira. Learning chemistry with VirtualLabs@Uma: a customizable 3D platform for new experimental protocols, Multimedia Tools and Applications, Volume 71 Issue 3, 1129-1155, (2014).

16. M.Muhamad, H.B. Zaman, H.B. and A. Ahmad, A. Developing virtual laboratory for biology (VLab-Bio): A proposed research conceptual framework. 2011 International Conference on Electrical Engineering and Informatics (ICEEI), Volume 1, 15-17 June, 1-6, (2011).

17. J. Lee, P. Quy, J. Kim, L. Kang ; A. Seo, K. HyungSeok, A Collaborative Virtual Reality Environment for Molecular Biology, Ubiquitous Virtual Reality, 2009. ISUVR '09. International Symposium on Ubiquitous Virtual Reality, 8-11 July, 68 - 71, (2009). 
18.K. Ahmed, A.N. Keeling, M. Fakhry, H. Ashrafian, R. Aggarwal, P. Naughton, A. Darzi, N. Cheshire, T. Athanasiou and M. Hamady, Role of virtual reality simulation in teaching and assessing technical skills in endovascular intervention. J Vasc Interv Radiol. 2010 Jan;21(1), 55-66, (2010).

19.H.W.R. Schreuder, G. Oei, M. Maas, J.C.C. Borleffs and M.P. Schijven, Implementation of simulation for training minimally invasive surgery. Tijdschrift voor Medisch Onderwijs, oktober 2011, Vol. 30, nr. 5, 206-220, (2011).

18. M. P. Guimarães and B. B. Gnecco. Teaching astronomy and celestial mechanics through virtual reality. Computer Applications in Engineering Education, 17(2), 196-205, (2009).

19. D. Dias, J. Brega, M. de Paiva Guimares, F. Modesto, B. Gnecco, and J. Lauris.3d semantic models for dental education. In M. Cruz-Cunha, J. Varajo, P. Powell, and R. Martinho, editors, ENTERprise Information Systems, volume 221 of Communications in Computer and Information Science, Springer Berlin Heidelberg, 89-96,(2011).

20. C. C. d. Souza, T. R. Medeiros, R. N. d. S. Gadelha, T. D. N. d. Sousa, E. L. Silva, and R. R. d. Azevedo. Um ambiente integrado de simulação para auxiliar o processo de ensino/aprendizagem da disciplina de sistemas operacionais. In Proceedings of Simpósio Brasileiro de Informática na Educação, 2011, 406-414, (2011).

21. L. Carozza, F. Bosche, and M. Abdel-Wahab. Robust 6-dof immersive navigation using commodity hardware. In Proceedings of the 20th ACM Symposium on Virtual Reality Software and Technology, VRST '14, New York, NY, USA, 2014. ACM, 19-22, (2014)

22. K. Beck. Embracing change with extreme programming. Computer, 32(10), Oct, 70-77, (1999).

23. K. Beck and C. Andres. Extreme Programming Explained: Embrace Change (2Nd Edition). Addison-Wesley Professional, (2004). 